\title{
Analytical electric field and sensitivity analysis for two microfluidic impedance cytometer designs
}

\author{
T. Sun, N.G. Green, S. Gawad and H. Morgan
}

\begin{abstract}
Microfabricated impedance cytometers have been developed to measure the electrical impedance of single biological particles at high speed. A general approach to analytically solve the electric field distributions for two different designs of cytometers: parallel facing electrodes and coplanar electrodes, using the Schwarz-Christoffel Mapping method is presented. Compared to previous analytical solutions, our derivations are more systematic and solutions are more straightforward. The solutions have been validated by comparison with numerical simulations performed using the finite element method. The influences on the electric field distribution due to the variations in the geometry of the devices have been discussed. A simple method is used to determine the impedance sensitivity of the system and to compare the two electrode designs. For identical geometrical parameters, we conclude that the parallel electrodes design is more sensitive than the coplanar electrodes.
\end{abstract}

\section{Introduction}

Microfluidic analytical systems have been developed over the last decade. Many of these have potential applications in the chemical, biochemical and medical fields. Examples of microfluidic systems include flow rate sensors [1,2], protein analysis systems [3] and particle manipulation tools [4-6]. One area of interest is high throughput microfluidic cytometers for single cell analysis. Several groups are developing different types of micro-flow cytometers [7-16]; devices that measure the optical and/or the electrical properties of single particles flowing in a microfluidic channel at high speed.

Impedance-based flow cytometers measure the dielectric properties of single particles $[8,10,13-16]$. The electrical properties of the particles are measured using microelectrodes, and two examples are shown schematically in Fig. 1. Two pairs of microelectrodes are fabricated within the microfluidic channel, one pair to measure the electrical properties of the particle suspended in the medium, and the other pair to measure the electrical properties of the medium only, which acts as reference. The electrodes are energised with a voltage at one or more discrete frequencies. When a particle passes over the electrodes, the differential signal between the pairs of electrodes is measured with a current to voltage converter and a differential amplifier to give the electrical impedance of the particle. For detailed description of the measurement system setup refer to Morgan et al. [16]. To detect the impedance signal from a single cell (e.g. red blood cell), the microelectrodes are fabricated with sizes similar to the cells, in the range 10-20 $\mu \mathrm{m}$.

(C) The Institution of Engineering and Technology 2007

doi:10.1049/iet-nbt:20070019

Paper first received 15th March and in revised form 20th June 2007

The authors are with Nanoscale Systems Integration Group, School of Electronics and Computer Science, University of Southampton, Highfield, Southampton SO17 1BJ, UK

S. Gawad is presently with LMIS4-STI-EPFL, Swiss Federal Institute of Technology, Lausanne 1015, Switzerland

E-mail: ts04r@ecs.soton.ac.uk
Fig. 1a shows a system with two pair of parallel facing electrodes while Fig. $1 b$ shows an alternative design with the electrodes fabricated only on the bottom of the channel. The latter is easier to fabricate but suffers from higher field non-uniformity. Because the electric field is not homogeneous, due to fringing fields, the impedance signal from the moving cell depends on the position of the cell within the detection volume. Therefore an accurate map of the electric field distribution in the device is required $a$ priori for evaluation of the impedance spectrum for a single cell.

The electric field depends on the geometry of the specific device, and can be determined using a number of methods, including charge density method [17], Green's theorem [18], Green's function [19], half-plane Green's function [20] and Fourier series [21,22], as well as numerically using for example finite element methods (FEMs) [23]. Schwarz-Christoffel Mapping (SCM) is a subset of conformal mapping and has been used to analyse electromagnetic field problems in MEMS devices, including coupling capacitance in comb-finger actuators [24], and forces in electromechanical actuators [25]. Of relevance to this paper are the techniques used to calculate the capacitance of a symmetrical-strip transmission line [26] and coplanarstrip waveguides $[27,28]$.

In a recent paper, SCM was used to calculate the resistance of a coplanar microfluidic impedance sensor [29]. The authors compared their theoretical calculations, particularly of the cell constant, with impedance measurements of aqueous solutions, and attributed discrepancies between experiment and theory to the fringing fields, non-zero electrode thickness and parasitic capacitance. However in their calculations, they failed to take into account the finite height of the channel, a point which was discussed by Linderholm and Renaud [30], who derived an improved analytical solution for the cell constant of the system. These authors applied a subsequent sine and bilinear transformation [30] to perform the conformal mapping, and derived an accurate value for the cell constant which gave good correlation with the 


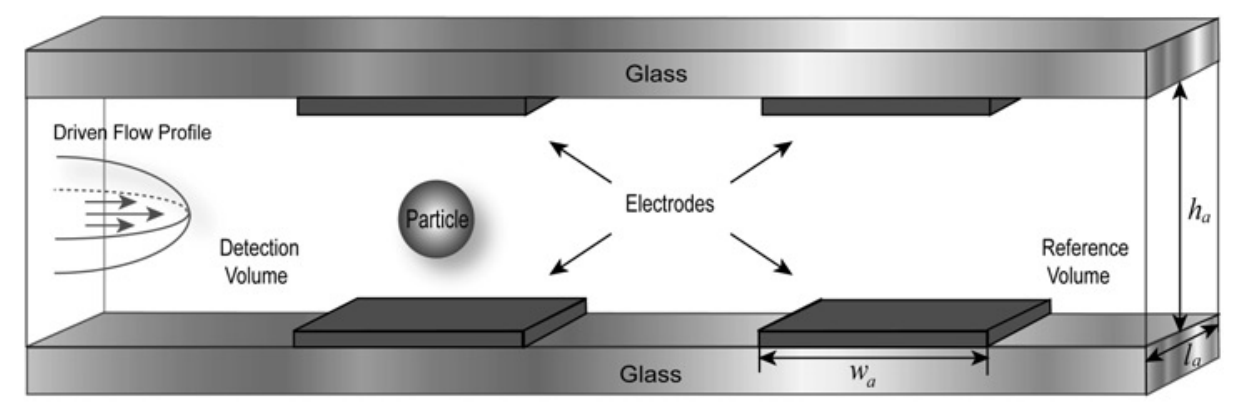

a

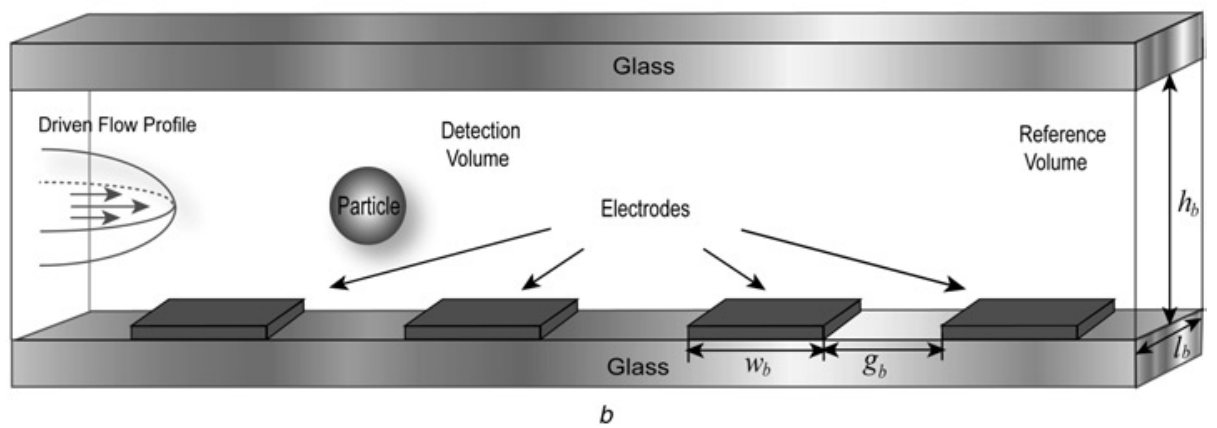

Fig. 1 Schematic diagram showing details of the microfluidic impedance cytometer, with two different designs of electrode configuration

In the device, the particles are suspended in an electrolyte and pass over the electrodes under an external hydrostatic pressure

$a$ Design A, the parallel facing electrode cytometer

Two pairs of electrodes are arranged to face each other; $w_{a}$ is the width of the electrodes; $l_{a}$ the length of the electrodes and $h_{a}$ the height of the channel

$b$ Design $\mathrm{B}$, the coplanar electrode cytometer

Electrodes are fabricated only on one side of the channel

These electrodes have width $w_{b}$, length $l_{b}$ with channel height $h_{b}$ and an electrode gap $g_{b}$

published experimental values of electrolyte conductivity. Later, Linderholm et al. [31] derived the analytical solution for electric field between two parallel facing electrodes using the similar procedure. More recently, Demierre et al. [32] utilised the method in the work of Linderholm and Renaud [30] to derive the electric field distribution in a micro-device for the dielectrophoretic manipulation of particles. However, the analytical methods used in these papers [30-32] are complicated and difficult to use. In particular, the bilinear transformation used by these authors makes the derivation procedure complicated and produces results which are difficult to interpret. The sine transformation can also be easily derived using SCM (see Section 7.1)

In this paper, we present a general method for the derivation of the electric field distribution for two different designs of micro-cytometers, based on SCM. Compared to the derivations [30-32], no sine transformation or bilinear transformation is needed, making the derivations systematic and straightforward. In the analytical solutions, the salient features of the electric field distribution can be clearly associated with the geometrical parameters of the cytometer. Inverse SCM transformations are performed to characterise the electric field lines in both cytometer designs. In order to validate the analytical solutions, the electric field distributions are numerically solved using the FEM, using FEMLAB 3.1 (Comsol Ltd). Comparison of the analytical solution and numerical simulation shows excellent agreement. Finally, we present an analytical model to evaluate the impedance sensitivity of the two designs, which takes the properties of the particle and medium in the system into account as well as the geometry of the device.

\section{SCM mapping}

The SCM method [33] maps the upper half of a complex plane ( $T$-plane) into the interior of a given polygon in another complex plane ( $Z$-plane). Let $\wp$ be an $m$-sided polygon in the $Z$-plane with vertices $Z_{1}, Z_{2}, \ldots, Z_{m}$ and interior angles $\theta_{1}, \theta_{2}, \ldots, \theta_{m}$, respectively. Along the real axis of the $T$-plane, $T_{1}, T_{2}, \ldots, T_{m}$ are the corresponding mapping points to $Z_{1}, Z_{2}, \ldots, Z_{m}$ in the $Z$-plane. The SCM integral, which maps the upper half of $T$-plane into the interior of $\wp$ in the $Z$-plane, is given by

$$
Z=C_{1} \int_{T_{0}}^{T} \prod_{r=1}^{m}\left(T-T_{r}\right)^{\left(\theta_{r} / \pi-1\right)} \mathrm{d} T+C_{2}
$$

where $C_{1}$ and $C_{2}$ are integral coefficients. $C_{1}$ establishes the scale and orientation of the polygon in the $Z$-plane and $C_{2}$ gives its position. $C_{1}$ and $C_{2}$ can be determined by the positions of the corresponding points in the $Z$ - and $T$-planes. The mapping system has three degrees of freedom which means up to three points $T_{r}(r=1,2,3)$ can be chosen arbitrarily along the real axis of $T$-plane. The point $T_{0}$ is the reference point, which is usually chosen as the zero point. Details of the SC mapping can found in [33].

For the electric field analysis, the real system, represented by an arbitrary two-dimensional polygon, is transformed into a rectangular region, equivalent to a parallel plate capacitor, where the electric field distribution is uniform. Generally, the standard technique consists of the following three steps

(i) Select a basic cell with two-dimensional geometry in the physical plane (Z-plane), with symmetrical axes. Determine 


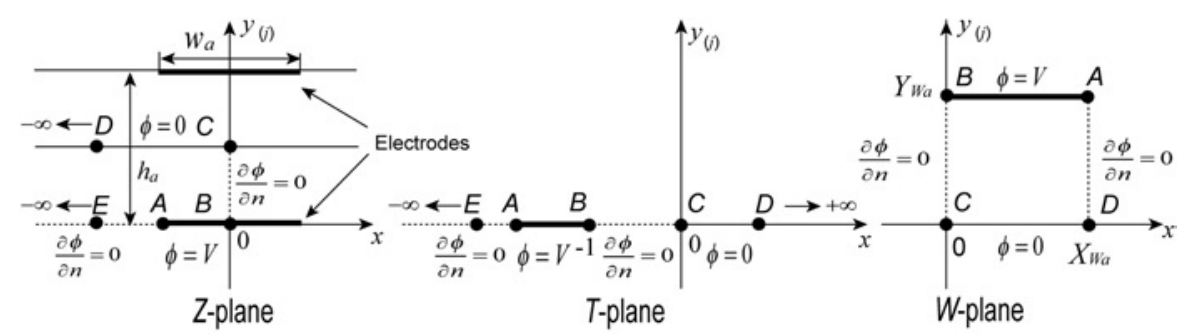

a

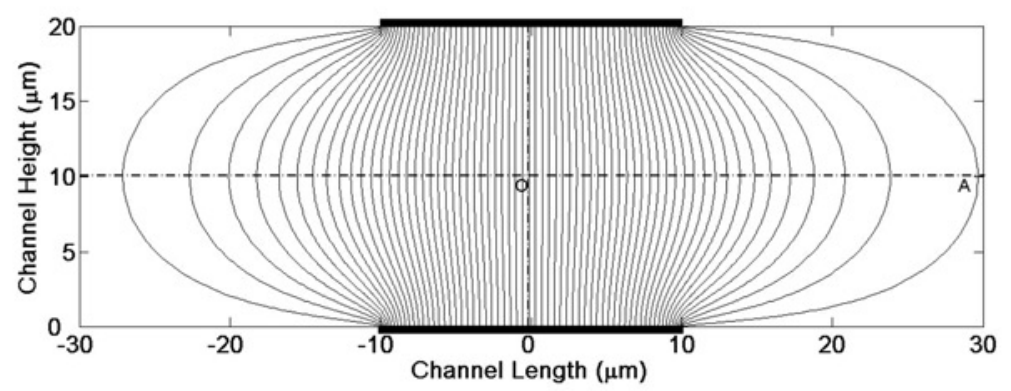

b

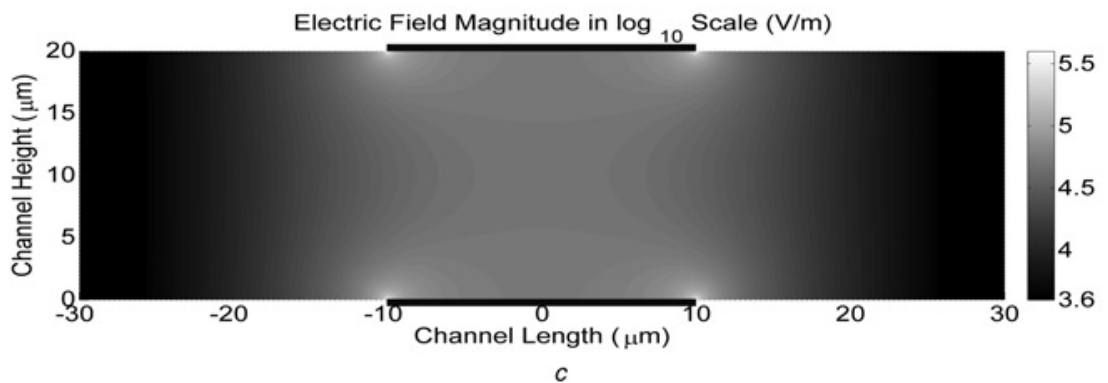

Fig. 2 Parallel facing electrode design

$a$ Diagrams showing the three different planes used for the parallel electrode geometry; physical plane (Z-plane); auxiliary plane (T-plane) and model plane ( $W$-plane)

$b$ Electric field lines for the parallel facing electrodes

Dotted lines are symmetry planes

Note that the channel extends to infinity on both sides

$c$ Magnitude of the electric field $(\mathrm{V} / \mathrm{m})$ for the parallel facing electrodes $\left(\log _{10}\right.$ scale $)$

the boundary conditions (i.e. Neumann or Dirichlet condition) along each boundary of the cell.

(ii) Apply SCM to open the selected basic cell and map it from the $Z$-plane to the upper half of the auxiliary plane (T-plane).

(iii) Apply a second SCM to transform the upper half of the $T$-plane into a closed parallel plate capacitor region in the model plane ( $W$-plane).

The non-uniform field problem in the $Z$-plane can then be easily solved in the $W$-plane. The detailed transformation procedure, together with the electric field analysis is shown in the next section.

\section{$3 \quad$ Electric field analysis}

The two electrode designs are shown in Fig. 1: Design A, parallel facing electrodes (Fig. 1a) and Design B, coplanar electrodes (Fig. 1b). In both designs, the electrodes are perpendicular to the direction of fluid flow in the channel, which has electrically insulating walls. The suspending medium is an electrolyte with fixed conductivity and permittivity. The boundary condition on the channel is insulating (zero current) and the electric field at all points is parallel to the side walls of the channel. Therefore the electric field distribution within the detection volume chamber can be considered two-dimensional.

\subsection{Design A parallel facing electrodes (subscript a)}

Fig. $2 a$ shows the planes used in the SCM for the parallel facing electrode design. The physical geometry in the $Z$-plane is mapped to the uniform electric field region in the $W$-plane via the auxiliary $T$-plane. Owing to symmetry, only one quarter of the geometry is solved, as indicated in the $Z$-plane and the thickness of the electrode is assumed to be zero. The mapping polygon has five points. $A, B$ and $C$ are fixed points, given by $Z_{A}=-w_{a} / 2, Z_{B}=0$ and $Z_{C}=j h_{a} / 2$, where $j$ is the imaginary unit $\left(j^{2}=-1\right), w_{a}$ is the width of the electrode and $h_{a}$ is the height of the channel. Points $D$ and $E$ go to infinity, and represent the semi-infinite channel. The Neumann boundary condition $\partial \phi / \partial n=0$, where $\phi$ is the potential and $n$ is the normal to the boundary, holds in the electrolyte at the interface between the bottom wall of the channel $(A E)$ and the boundary at the right $(B C)$, as shown by the dashed lines in the Fig. 2a. Dirichlet boundary conditions define the fixed potential $\phi=V$ on the electrode $(A B)$ and the boundary for the axis of odd symmetry, $\phi=0(C D)$.

In the auxiliary plane ( $T$-plane), the whole polygon $A B C D E$ is mapped into the upper half of the $T$-plane and 
all the boundaries are mapped onto the real axis. The corresponding points are: $T_{A}, T_{B}$ and $T_{C}$. The points $D$ and $E$ are mapped to infinity. Since SCM method allows that up to three points can be chosen arbitrarily along the real axis of $T$-plane, we fixed the coordinates of $T_{B}=-1, T_{C}=0$. The two interior angles of polygon $A B C D E$ at the points $B$ and $C$ are both $\pi / 2$. The SCM integral from the $T$-plane to the $Z$-plane is given by

$$
Z=C_{1} \int^{T}\left(T-T_{B}\right)^{-1 / 2}\left(T-T_{C}\right)^{-1 / 2} \mathrm{~d} T+C_{2}
$$

where $Z=Z_{x}+j Z_{y}$ refers to the complex coordinate of any point in the interior of polygon $A B C D E$ in $Z$-plane. $T=T_{x}+j T_{y}$ refers to the complex coordinate of any point in the upper half of $T$-plane.

The solution of (2) is given by

$$
Z=2 C_{1} \ln (\sqrt{T}+\sqrt{T+1})+C_{2}
$$

Equation (3) links the $T$-plane with the $Z$-plane. The values of the coefficients $C_{1}$ and $C_{2}$ can be solved by a mapping relationship between the coordinates of the corresponding points in the two planes.

For coordinate point $B, T=T_{B}$, (3) becomes

$$
Z_{B}=j \pi C_{1}+C_{2}=0
$$

For coordinate point $C, T=T_{C}$, (3) becomes

$$
Z_{C}=C_{2}=j \frac{h_{a}}{2}
$$

Substituting (4) and (5) into (3), (3) becomes

$$
Z=\frac{-h_{a}}{\pi} \ln (\sqrt{T}+\sqrt{T+1})+\frac{j h_{a}}{2}
$$

Equation (6) is the final solution for the transformation from $T$-plane to Z-plane, the same result as in [26]. The inverse function of (6) allows $T$ to be expressed as a function of $Z$

$$
T=\sinh ^{2}\left[\frac{\pi}{h_{a}}\left(Z-\frac{j h_{a}}{2}\right)\right]
$$

The coordinate of point $A$ in the $T$-plane can be obtained from (7)

$$
T_{A}=-\cosh ^{2}\left(\frac{\pi w_{a}}{2 h_{a}}\right)
$$

The second SCM is used to transform the upper half of the $T$-plane into a rectangle in the model plane ( $W$-plane). The electric field is uniformly distributed in the interior of the rectangle, due to the restriction from the transformed boundaries in $W$-plane. The corresponding points are: $W_{A}=X_{W a}+j Y_{W a}, W_{B}=j Y_{W a}, W_{C}=0$ and $W_{D}=X_{W a}$, where $X_{W a}$ and $Y_{W a}$ are the size of the rectangle along the real and imaginary axis, respectively. Similarly, the transformation from the $T$-plane to the $W$-plane is given by

$$
\begin{aligned}
W= & D_{1} \int^{T}\left(T-T_{C}\right)^{-1 / 2}\left(T-T_{B}\right)^{-1 / 2}\left(T-T_{A}\right)^{-1 / 2} \mathrm{~d} T \\
& +D_{2}
\end{aligned}
$$

For $T>T_{E}>T_{B}>T_{A}$, the solution of (9) is an elliptic integral [34]

$$
\begin{aligned}
W & =D_{3} F\left(\varpi_{a}, k_{a}\right)+D_{2} \\
& =D_{3} \int_{0}^{\lambda_{a}} \frac{\mathrm{d} \lambda_{a}}{\left(1-\lambda_{a}^{2}\right)\left(1-k_{a}^{2} \lambda_{a}^{2}\right)} \mathrm{d} \lambda_{a}+D_{2}
\end{aligned}
$$

with

$$
\begin{aligned}
& D_{3}=\frac{2 D_{1}}{\sqrt{T_{C}-T_{A}}}, \quad \varpi_{a}=\arcsin \left(\sqrt{\frac{T-T_{C}}{T-T_{B}}}\right), \\
& k_{a}=\sqrt{\frac{T_{B}-T_{A}}{T_{C}-T_{A}}}, \quad \lambda_{a}=\sin \varpi_{a}
\end{aligned}
$$

where $F\left(\varpi_{a}, k_{a}\right)$ is the elliptical integral of the first kind and $k_{a}$ is the modulus of the elliptic function.

Equation (10) links the $T$-plane with the $W$-plane. The values of the coefficients $D_{2}$ and $D_{3}$ can be obtained by mapping the coordinates of the corresponding points in $T$ - and $W$-plane.

For coordinate point $C, T=T_{C}$, therefore $\lambda_{a}=0$ and (10) becomes

$$
W_{C}=D_{2}=0
$$

For coordinate point $B, T=T_{B}$, therefore $\lambda_{a}=\infty$ and (10) becomes

$$
\begin{aligned}
W_{B} & =D_{3} \int_{0}^{\infty} \frac{\mathrm{d} \lambda_{a}}{\left(1-\lambda_{a}^{2}\right)\left(1-k_{a}^{2} \lambda_{a}^{2}\right)} \mathrm{d} \lambda_{a}=D_{3} j K\left(k_{a}^{\prime}\right) \\
& =j Y_{W a}
\end{aligned}
$$

For coordinate point $A, T=T_{A}$ therefore $\lambda_{a}=1 / k_{a}$ and (10) becomes

$$
\begin{aligned}
W_{A} & =D_{3} \int_{0}^{1 / k_{a}} \frac{\mathrm{d} \lambda_{a}}{\left(1-\lambda_{a}^{2}\right)\left(1-k_{a}^{2} \lambda_{a}^{2}\right)} \mathrm{d} \lambda_{a} \\
& =D_{3}\left[K\left(k_{a}\right)+j K\left(k_{a}^{\prime}\right)\right]=X_{W a}+j Y_{W a}
\end{aligned}
$$

Combining (12) and (13), $D_{3}$ is given by

$$
D_{3}=\frac{Y_{W a}}{K\left(k_{a}^{\prime}\right)}=\frac{X_{W a}}{K\left(k_{a}\right)}
$$

where $K\left(k_{a}\right)$ and $K\left(k_{a}^{\prime}\right)$ are the complete elliptic integral of the first kind with $k_{a}^{\prime}=\sqrt{\left(1-k_{a}^{2}\right)}$.

The cell constant $[29,30]$ per unit width of the microfluidic channel in Design A used for evaluating the impedance is given by

$$
\kappa_{a}=\frac{X_{W a}}{Y_{W a}}=\frac{K\left(k_{a}\right)}{K\left(k_{a}^{\prime}\right)}
$$

By solving the inverse function of (10), we use $W$ to express $T$

$$
T=\frac{T_{C}-T_{B} \operatorname{sn}^{2}\left(W / D_{3}, k_{a}\right)}{\operatorname{cn}^{2}\left(W / D_{3}, k_{a}\right)}
$$

where $\operatorname{sn}(\ldots, \ldots)$ and $\operatorname{cn}(\ldots, \ldots)$ are the Jacobian elliptic functions. 
Substituting (16) into (6), we obtain a direct relationship between the $W$-plane and $Z$-plane

$$
\begin{aligned}
Z= & \frac{-h_{a}}{\pi} \ln \left(\left(\frac{T_{C}-T_{B} \operatorname{sn}^{2}\left(W / D_{3}, k_{a}\right)}{\operatorname{cn}^{2}\left(W / D_{3}, k_{a}\right)}\right)^{1 / 2}\right. \\
& \left.+\left(\frac{T_{C}-T_{B} \operatorname{sn}^{2}\left(W / D_{3}, k_{a}\right)}{\operatorname{cn}^{2}\left(W / D_{3}, k_{a}\right)}+1\right)^{1 / 2}\right)+\frac{j h_{a}}{2}
\end{aligned}
$$

Equation (17) provides an easy and convenient way to derive the distribution of electric field lines between the two electrodes. In the $W$-plane, where the electric field is uniform, every electric field line is perpendicular to the electrode $(A B)$. Therefore every point on a specific electric field line has the same coordinate on the real axis. In this case, if we choose one group of points which share the same real axis coordinate (one electric field line in $W$-plane) and use (17) to convert the coordinates of all these points in the $W$-plane to the $Z$-plane, we derive the corresponding electric field line in the $Z$-plane. Fig. $2 b$ shows the electric field lines for Design A. The electrodes are shown in the figure (and also the following figures).

The complex variable calculations for the Jacobian Elliptic Functions can be separated into real and imaginary components using the following formulae [35].

$$
\begin{aligned}
\operatorname{sn}(u+j v, k)= & \frac{\operatorname{sn}(u, k) \operatorname{dn}\left(v, k^{\prime}\right)}{1-\operatorname{sn}^{2}\left(v, k^{\prime}\right) \operatorname{dn}^{2}(u, k)} \\
& +j \frac{\operatorname{cn}(u, k) \operatorname{dn}(u, k) \operatorname{sn}\left(v, k^{\prime}\right) \operatorname{cn}\left(v, k^{\prime}\right)}{1-\operatorname{sn}^{2}\left(v, k^{\prime}\right) \operatorname{dn}^{2}(u, k)} \\
\operatorname{cn}(u+j v, k)= & \frac{\operatorname{cn}(u, k) \operatorname{cn}\left(v, k^{\prime}\right)}{1-\operatorname{sn}^{2}\left(v, k^{\prime}\right) \operatorname{dn}^{2}(u, k)} \\
& +j \frac{\operatorname{sn}(u, k) \operatorname{dn}(u, k) \operatorname{sn}\left(v, k^{\prime}\right) \operatorname{dn}\left(v, k^{\prime}\right)}{1-\operatorname{sn}^{2}\left(v, k^{\prime}\right) \operatorname{dn}^{2}(u, k)}
\end{aligned}
$$

where $\operatorname{sn}(\ldots, \ldots), \operatorname{cn}(\ldots, \ldots)$ and $\operatorname{dn}(\ldots, \ldots)$ are the Jacobian elliptic functions.

Since Laplace's equation remains invariant under conformal mapping, the relationship linking the potential gradients in the physical plane and model plane is [33]

$$
\nabla \phi_{Z}=\nabla \phi_{W} \overline{f^{\prime}(Z)}=\nabla \phi_{W} \frac{\overline{\mathrm{d} W}}{\mathrm{~d} Z}
$$

where, $\nabla \phi_{Z}$ and $\nabla \phi_{W}$ are the potential gradients in $Z$-plane and $W$-plane, respectively. $\overline{f^{\prime}(Z)}$ is the conjugate of the derivative of $f(Z)$, which is the linking transformation equation between the $Z$-plane and the $W$-plane.

Utilising the relationship in (19) and combing (2) and (9), the non-uniform electric field distribution in the $Z$-plane can be easily derived as

$$
\begin{aligned}
& \boldsymbol{E}_{Z a}=-\nabla \phi_{Z a}=-\nabla \phi_{W a} \overline{\left(\frac{\mathrm{d} W}{\mathrm{~d} T} \frac{\mathrm{d} T}{\mathrm{~d} Z}\right)}
\end{aligned}
$$

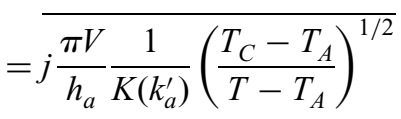

where $\boldsymbol{E}_{Z a}$ is the electric field distribution in the $Z$-plane for Design A. $\phi_{Z a}$ and $\phi_{W a}$ are the potential distributions in the $Z$-plane and $W$-plane, respectively. $V$ is the potential difference between the electrodes $A B$ and the axis of odd symmetry, $C D$. The value of $T_{A}$ is given by (8) and $T_{C}$ is chosen at the origin.
Equation (20) is the analytical solution for the electric field, Design A. Compared to the previous analytical solution [31], the salient features of field distribution are easier to identify. The magnitude of the electric field goes to infinity at point $A$ (when $T=T_{A}$ ), that is the electrode edge. Numerical calculations (20) were used to plot the field distribution using MATLAB ${ }^{\mathrm{TM}}$ (Mathworks Inc. USA). Fig. $2 c$ shows a 2D plot of the magnitude of the electric field for Design A. The field magnitude increases sharply towards the edges of the electrodes, the fringing field. In the central region between the two facing electrodes, the electric field magnitude is quasi-homogeneous.

\subsection{Design B coplanar electrodes (subscript b)}

Fig. $3 a$ shows a representation of the configuration of coplanar electrodes in the $Z$-, $T$ - and $W$ - planes. From symmetry, only half the channel is analysed, and the polygonal region in the $Z$-plane has six points: $A, B, C$ and $D$, given by $Z_{A}=w_{b}+g_{b} / 2, Z_{B}=g_{b} / 2, Z_{C}=0$ and $Z_{D}=j h_{b}$, and $E$, $F$ tend to infinity. Again, $w_{b}$ is the width of the electrodes, $h_{b}$ is the height of the channel and $g_{b}$ is the gap separating the two electrodes. Neumann boundary conditions $\partial \phi / \partial n=0$ hold on boundaries $A F, B C$ and $D E$ (indicated by dashed lines) and Dirichlet boundary conditions hold on the electrode $A B(\phi=V)$ and the boundary $C D(\phi=0)$ (indicated by solid lines). The corresponding points in the $T$-plane are: $T_{A}, T_{B}, T_{C}=1$ and $T_{D}=0$, and $E, F$ tend to infinity. The interior polygon angles in $Z$-plane at $C$ and $D$ are both $\pi / 2$. From the SCM integral formula, the transformation from $Z$-plane to $T$-plane is

$$
Z=C_{1} \int^{T}\left(T-T_{C}\right)^{-1 / 2}\left(T-T_{D}\right)^{-1 / 2} \mathrm{~d} T+C_{2}
$$

Following the procedure used for Design A, the solution of (21) is

$$
Z=\frac{2 h_{b}}{\pi} \ln (\sqrt{T}+\sqrt{T-1})
$$

The inverse function of (22) is

$$
T=\cosh ^{2}\left(\frac{\pi Z}{2 h_{b}}\right)
$$

The values of $T_{A}$ and $T_{B}$ are

$$
T_{A}=\cosh ^{2}\left(\frac{\pi\left(g_{b}+2 w_{b}\right)}{4 h_{b}}\right), \quad T_{B}=\cosh ^{2}\left(\frac{\pi g_{b}}{4 h_{b}}\right)
$$

In the model plane ( $W$-plane), the original polygon is transformed into a rectangle, where the electric field distribution is uniform. The corresponding points are: $W_{A}=0$, $W_{B}=j Y_{W b}, W_{C}=X_{W b}+j Y_{W b}$ and $W_{D}=X_{W b}$. Again, the SCM integral from $T$-plane to $W$-plane is given by

$$
\begin{aligned}
W= & D_{1} \int^{T}\left(T-T_{A}\right)^{-1 / 2}\left(T-T_{B}\right)^{-1 / 2} \\
& \times\left(T-T_{C}\right)^{-1 / 2}\left(T-T_{D}\right)^{-1 / 2} \mathrm{~d} T+D_{2}
\end{aligned}
$$

For $T>T_{A}>T_{B}>T_{C}>T_{D}$, the solution of (25) is an elliptic integral [34]

$$
\begin{aligned}
W & =D_{3} F\left(\varpi_{b}, k_{b}\right)+D_{2} \\
& =D_{3} \int_{0}^{\lambda_{b}} \frac{\mathrm{d} \lambda_{b}}{\left(1-\lambda_{b}^{2}\right)\left(1-k_{b}^{2} \lambda_{b}^{2}\right)} \mathrm{d} \lambda_{b}+D_{2}
\end{aligned}
$$


with

$$
\begin{aligned}
D_{3} & =\frac{2 D_{1}}{\sqrt{\left(T_{A}-T_{C}\right)\left(T_{B}-T_{D}\right)}}, \\
\varpi_{b} & =\arcsin \left(\sqrt{\frac{\left(T_{B}-T_{D}\right)\left(T-T_{A}\right)}{\left(T_{A}-T_{D}\right)\left(T-T_{B}\right)}}\right) \\
k_{b} & =\sqrt{\frac{\left(T_{B}-T_{C}\right)\left(T_{A}-T_{D}\right)}{\left(T_{A}-T_{C}\right)\left(T_{B}-T_{D}\right)}}, \quad \lambda_{b}=\sin \varpi_{b}
\end{aligned}
$$

The values of the coefficients $D_{2}$ and $D_{3}$ are obtained from the corresponding coordinates of points $A, B, C$ and $D$ in $T$ and $W$-plane.

$$
D_{2}=0 \quad D_{3}=\frac{Y_{W b}}{K\left(k_{b}^{\prime}\right)}=\frac{X_{W b}}{K\left(k_{b}\right)}
$$

As introduced in Section (3.1), $K\left(k_{b}\right)$ is the complete elliptic integral of the first kind and $k_{b}$ is the modulus of the elliptic function which is different in this case. The cell constant per unit width of microfluidic channel in Design $\mathrm{B}$ is given by

$$
\kappa_{b}=\frac{Y_{W b}}{2 X_{W b}}=\frac{K\left(k_{b}^{\prime}\right)}{2 K\left(k_{b}\right)}
$$

In this case, the result is multiplied by 2 since only half of the microchannel is mapped.

In the recent paper by Hong et al. [29], the cell constant of Design B was sub-divided into a resistive $\left(1 / \kappa_{b}\right)$ and capacitive cell constant $\left(\kappa_{b}\right)$. However, in their conformal mapping analysis, the authors ignored the finite height of the channel, causing significant deviation between experimental results and theoretical calculations. Using a sine and bilinear transformation, Linderholm and Renaud [30] published the correct cell constant and showed a good fit between theory and experiment, assuming the conductivity of the DI water in Hong et al.'s [29] experiments to be $20 \mu \mathrm{S} / \mathrm{cm}$. We have performed a similar analysis using the SCM method and find excellent agreement with Hong et al.'s corrected data (see Section 7.2)

The inverse function of (26) is

$$
T=\frac{T_{A} T_{B} \operatorname{cn}^{2}\left(W / D_{3}, k_{b}\right)}{T_{B}-T_{A} \operatorname{sn}^{2}\left(W / D_{3}, k_{b}\right)}
$$

Substituting (29) into (22), the direct transformation from $W$-plane to $Z$-plane is

$$
\begin{aligned}
Z= & \frac{2 h_{b}}{\pi} \ln \left(\left(\frac{T_{A} T_{B} \mathrm{cn}^{2}\left(W / D_{3}, k_{b}\right)}{T_{B}-T_{A} \operatorname{sn}^{2}\left(W / D_{3}, k_{b}\right)}\right)^{1 / 2}\right. \\
& \left.+\left(\frac{T_{A} T_{B} \mathrm{cn}^{2}\left(W / D_{3}, k_{b}\right)}{T_{B}-T_{A} \operatorname{sn}^{2}\left(W / D_{3}, k_{b}\right)}-1\right)^{1 / 2}\right)
\end{aligned}
$$

The distribution of the electric field (Fig. $3 b$ ) is derived using the same principle as for Design A, using (30). The only difference is that in Design $\mathrm{B}$, all points on one electric field line share the same imaginary axis coordinate.

Employing the relationship linking the potential gradients in the physical plane and model plane (19), the electric field

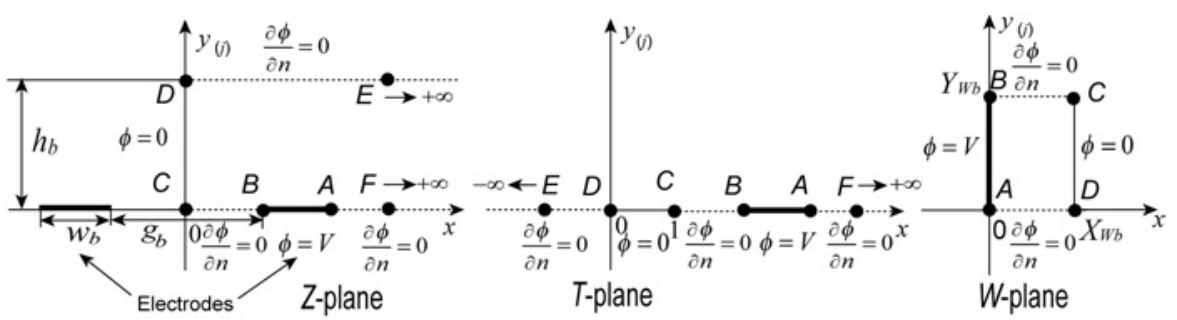

a
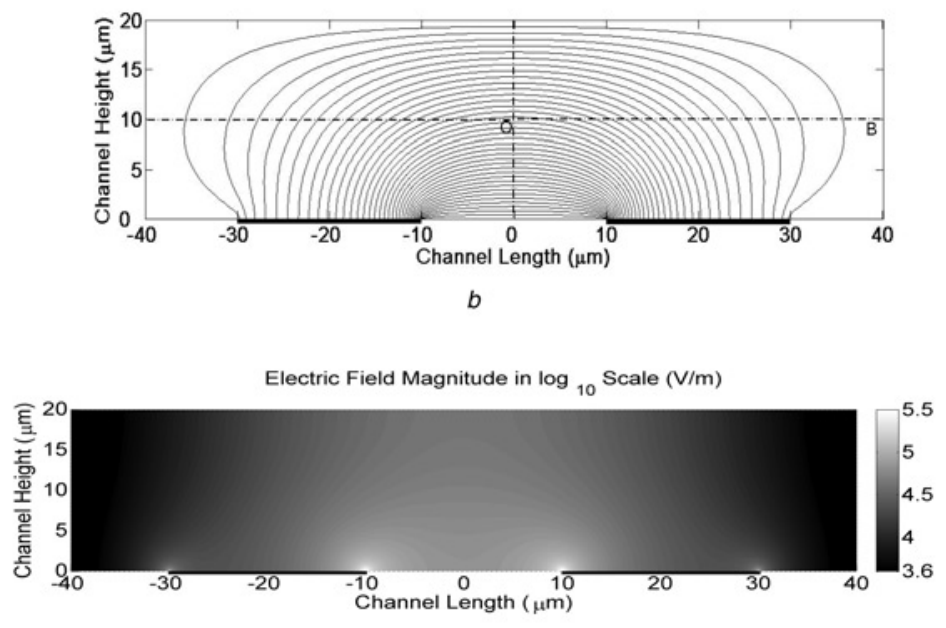

c

Fig. 3 Coplanar electrode design

$a$ Diagrams showing the three different planes for the coplanar electrode arrangement: physical plane ( $Z$-plane); auxiliary plane ( $T$-plane) and model plane $(W$-plane $)$

$b$ Electric field lines for co-planar electrodes

Dotted lines are symmetry planes

Note that the channel extends to infinity on both sides

$c$ Magnitude of the electric field $(\mathrm{V} / \mathrm{m})$ for the coplanar electrodes ( $\log _{10}$ scale) 
in Design B is

$$
\boldsymbol{E}_{Z b}=\overline{\frac{\pi V}{2 h_{b} K\left(k_{b}\right)}\left(\frac{T_{A}-T_{C}}{T-T_{A}}\right)^{1 / 2}\left(\frac{T_{B}-T_{D}}{T-T_{B}}\right)^{1 / 2}}
$$

In this expression, $V$ is the potential difference between electrode $A B$ and the axis of odd symmetry, $C D$. The values of $T_{A}$ and $T_{B}$ are given by (24). $T_{C}$ is chosen to be $1, T_{D}$ is chosen to be the origin.

Equation (31) is the analytical solution for the electric field for Design B; again the electric field distribution is easier to analyse, going to infinity at points $A$ and $B$ (when $T=T_{A}$ or $T=T_{B}$ ), at the electrode edges. The magnitude of the electric field for design $\mathrm{B}$ is plotted in Fig. $3 c$ and shows that the electric field for this design is quasihomogeneous in the gap between the two electrodes. In terms of analysis of cells, the field changes by only a small amount along the channel axis, either side of the central symmetry line.

\subsection{Comparisons with FEM}

The analytical solutions were compared with numerical simulations performed using the FEM in FEMLAB ${ }^{\mathrm{TM}}$ (Comsol Ltd). From symmetry, comparisons are made along a central horizontal line through half of the channel; OA for Design A and OB for Design B (Fig. $2 b$ and $3 b$ ).

3.3.1 Design A: The electric field distribution depends on two geometrical parameters: the electrode width and channel height. Fig. $4 a$ shows the results for fixed channel height and variable electrode width, while in Fig. $4 b$ the width of the
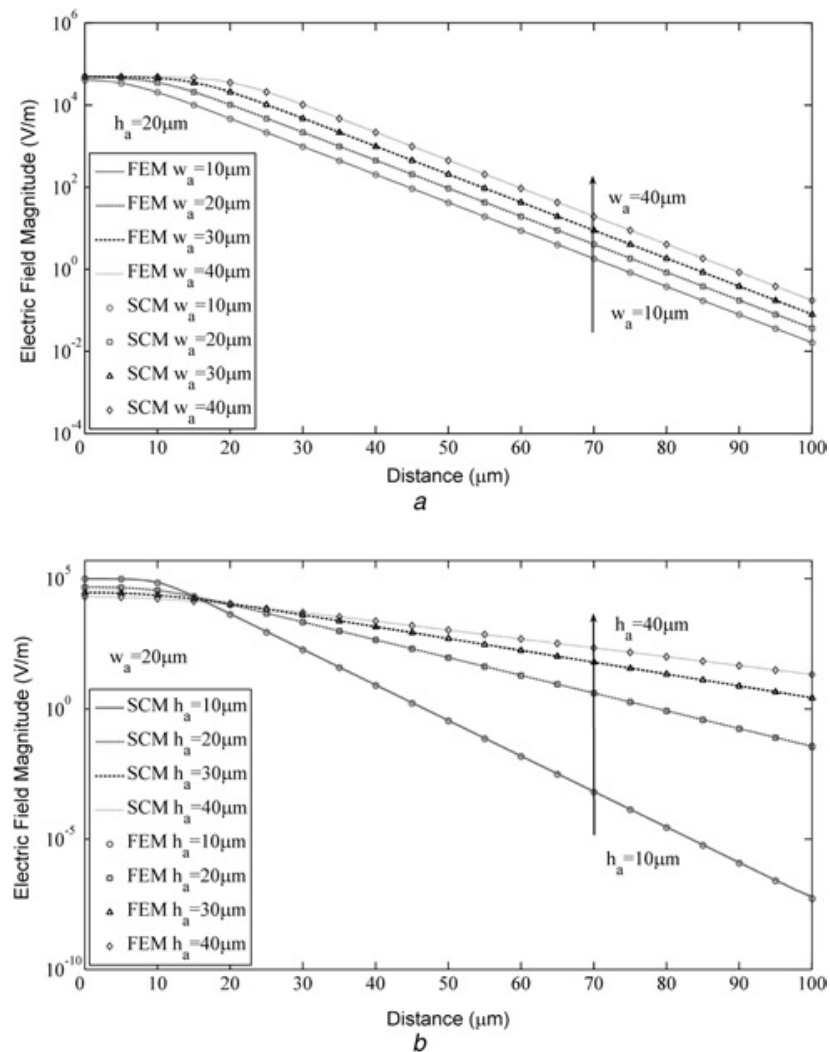

Fig. 4 Electric field magnitude plotted against distance from the centre of the electrode along the channel axis (OA) for Design A Lines are data from SCM, symbols are data from FEMLAB (FEM) $a$ Height of the channel and the gap is fixed at $20 \mu \mathrm{m}$; the width of the electrode is varied from 10 to $40 \mu \mathrm{m}$

$b$ Width of the electrode is fixed at $20 \mu \mathrm{m}$ and the channel height varied from 10 to $40 \mu \mathrm{m}$ electrode is fixed and channel height varied. Excellent agreement is observed between the SCM and FEM in both cases. Fig. $4 a$ shows that as the electrode width increases (with fixed channel height), the quasi-homogeneous electric field region becomes wider and the magnitude of the electric field in the entire microchannel increases. Fig. $4 b$ shows that as the channel height increases (with fixed electrode width), the magnitude of the field in the quasi-homogeneous region decreases. However, the field magnitude outside the quasi-homogeneous region increases.

3.3.2 Design B: The electric field distribution depends on three geometrical parameters: the electrode width, channel height and the gap between the electrodes. Fig. $5 a$ compares
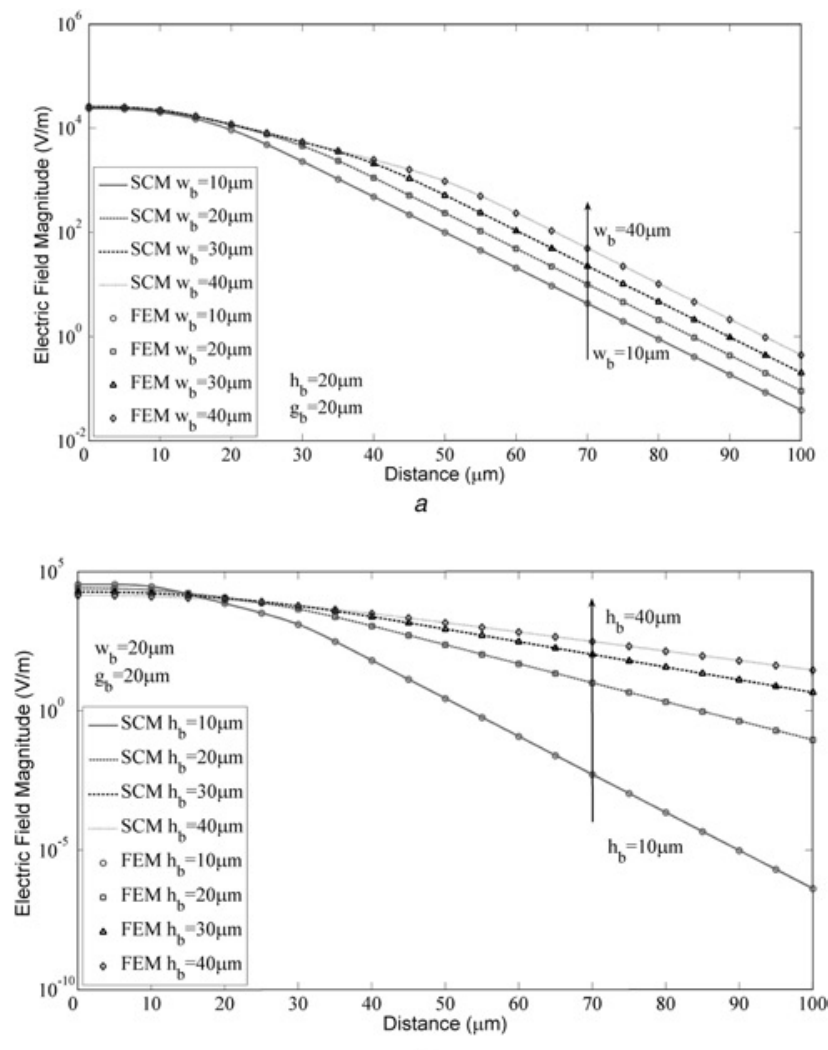

$b$

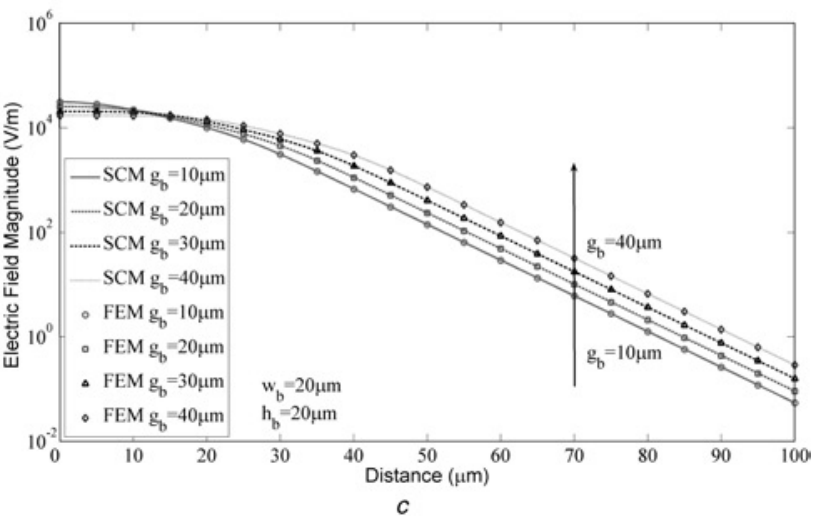

Fig. 5 Electric field magnitude plotted against distance from the centre of the gap between the electrodes, along the channel axis (OB) for Design $B$

Lines are date from SCM, symbols are FEMLAB (FEM) data $a$ Height of the channel and the gap is fixed at $20 \mu \mathrm{m}$, the electrode width is varied from 10 to $40 \mu \mathrm{m}$

$b$ Width of the electrode and gap is fixed at $20 \mu \mathrm{m}$ and the height of the channel is varied from 10 to $40 \mu \mathrm{m}$

$c$ Height of the channel and electrode width is fixed at $20 \mu \mathrm{m}$ and the gap is varied from 10 to $40 \mu \mathrm{m}$ 
the two methods for fixed channel height and electrode gap, and variable electrode width. In Fig. $5 b$ the electrode width and gap are fixed and the channel height is varied while Fig. $5 c$ the channel height and electrode width is fixed and the gap is varied. Again, the agreements between the SCM and FEM are excellent in all three cases. The effect of changing channel height is similar to Design $\mathrm{A}$, however, increasing the electrode width does not influence the region of quasi-homogeneous electric field (in the centre). Increasing the electrode gap leads to a larger region of quasi-homogeneous field in the centre, (but decreases the magnitude) and increases the magnitude of the electric field outside the inter-electrode region.

3.3.3 SCM and FEM: Figs. 4 and 5 both show that the SCM solutions and FEM numerical solutions are in excellent agreement, where the typical deviation is $<0.5 \%$, due to the numerical error. The main advantages of SCM solutions can be identified from adaptive geometrical changes, accuracy, computational time and guidance for device design.

The SCM solution for Design A (20) and Design B (31) directly give the electric field distribution for any value of geometrical parameter (electrode width, channel height or gap distance) of the device. In contrast, a different FEM simulation has to be performed for any arbitrary geometrical parameter. Also the accuracy of FEM solutions is influenced by the mesh conditions; a large number of refined mesh elements are required for an accurate solution. In order to improve the efficiency of the FEM simulations, while maintaining the accuracy, we increased the mesh density only on the electrode edges and along the paths where the values of the electric field are sampled. The computational convergence in FEMLAB was set to $10^{-6}$; each simulation used approximately 76800 mesh elements and the computational time was $21.4 \mathrm{~s}$ ( $\mathrm{P} 4$ computer, $3 \mathrm{G} \mathrm{Hz}$ CPU, 2 GB RAM). The time taken to calculate the SCM solution (MATLAB, with spatial resolution set to $0.1 \mu \mathrm{m}$ ) was $0.46 \mathrm{~s}$, much faster than FEM simulations.

\subsection{Impedance sensitivity}

The sensitivity of Design A (parallel facing electrodes) has been discussed by Linderholm et al. [31]. These authors defined the sensitivity as the ratio of local power dissipation to total power dissipated in the medium, effectively related to the electric field magnitude squared and the cell constant of the device. They then proposed an optimum ratio of electrode width and channel height $(w / h \simeq 0.56)$ (see Section 7.3)

We present an alternative method to evaluate the sensitivity, $S$ of the system, defined as the relative change in impedance

$$
S=\frac{|\Delta \tilde{Z}|}{\left|\tilde{Z}_{m}\right|}=\frac{|| \tilde{Z}_{\text {mix }}|-| \tilde{Z}_{m}||}{\left|\tilde{Z}_{m}\right|}
$$

where $\Delta \tilde{Z}$ is the impedance change due to the presence of a particle, $\tilde{Z}_{m}$ is the complex impedance of the detection volume containing medium and $\tilde{Z}_{\text {mix }}$ is the complex impedance of the mixture (the medium and the particle ) in the detection volume.

This method not only considers the geometry of the system, but also includes the dielectric properties of the particle and medium, and the volume fraction. In conventional dielectric spectroscopy, the dielectric properties of the suspending mixture is calculated using Maxwell's Mixture Theory (MMT) [36], which relates the complex permittivity of the mixture to the volume fraction, and the complex permittivity of the particle and the suspending medium. MMT can be used to calculate the impedance of particles in a homogeneous electric field and the volume fraction is low $(<20 \%)$. Therefore the sensitivity function is only valid for a small particle located in the centre of the system, where the electric field is quasi-homogenous, as shown in
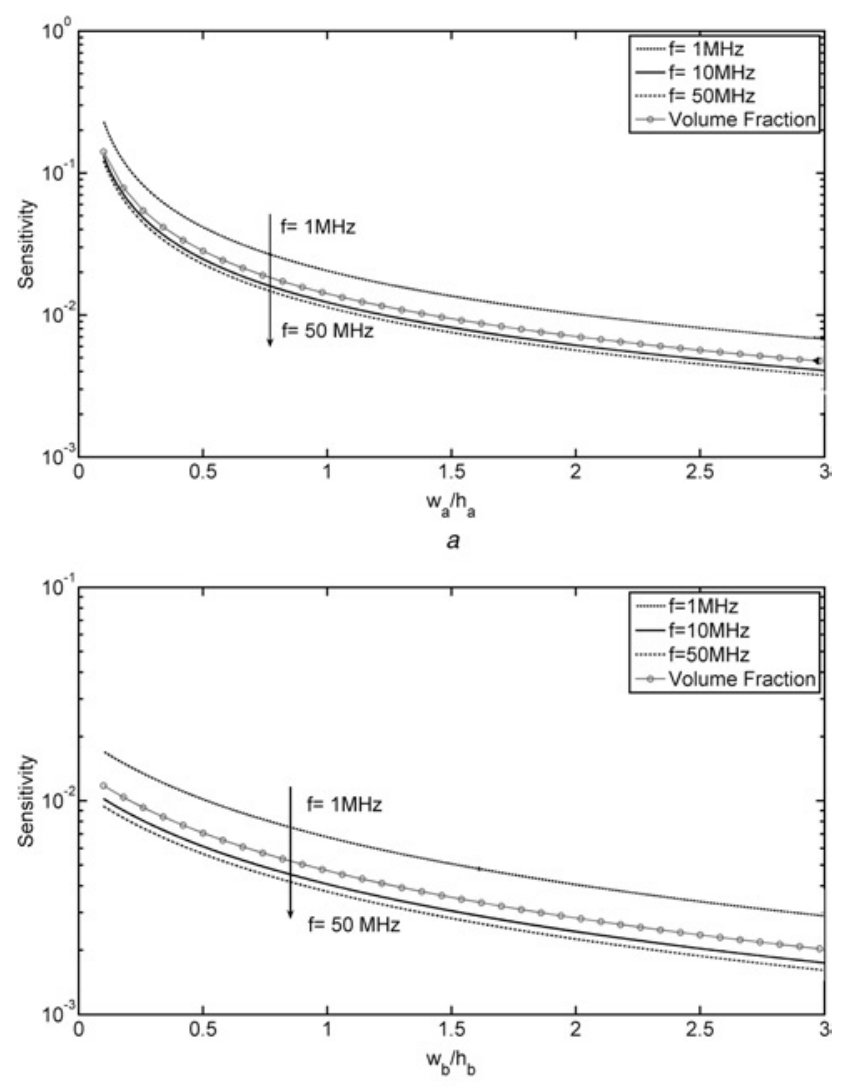

$b$

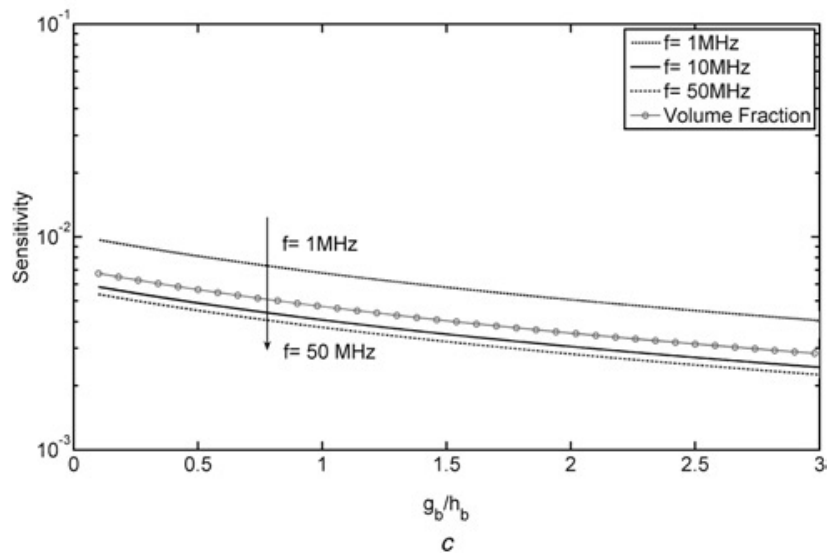

Fig. 6 Plot of the sensitivity $\left(|\Delta \tilde{Z}| /\left|\tilde{Z}_{m}\right|\right)$ for different electrode geometries at three discrete frequencies

Variation in the volume fraction in each case is plotted in the corresponding sub-figure

Data was calculated with the following parameters: $\varepsilon_{o}=8.854 \times$ $10^{-12} \mathrm{Fm}^{-1}$, cell radius: $R=3 \times 10^{-6} \mathrm{~m}$, thickness of cell membrane: $d=5 \times 10^{-9} \mathrm{~m}$, permittivity of medium: $\varepsilon_{m}=80 \times \varepsilon_{o}$, conductivity of medium: $\sigma_{m}=1.6 \mathrm{Sm}^{-1}$, permittivity of cell membrane: $\varepsilon_{\text {mem }}=5 \times \varepsilon_{o}$, conductivity of cell membrane: $\sigma_{\text {mem }}=10^{-8} \mathrm{Sm}^{-1}$, permittivity of cell cytoplasm: $\varepsilon_{i}=60 \times \varepsilon_{o}$, conductivity of cell membrane: $\sigma_{i}=0.6 \mathrm{Sm}^{-1}$

$a$ Design $\mathrm{A}$, for different ratios of electrode width to the channel height

$b$ Design $\mathrm{B}$, for different ratios of electrode width to the channel height, with fixed gap $(20 \mu \mathrm{m})$

$c$ Design B, for different ratios of gap to the channel height, with fixed electrode width $(20 \mu \mathrm{m})$ 
Fig. 1. The volume fraction is difficult to determine precisely because of the fringing field. In order to calculate the sensitivity, we define the volume of the detection area as the perimeter of the electrodes and the height of the channel. The complex impedance of the mixture and medium were calculated as shown in [16] using the cell constant $(\kappa)$ of the device to consider the contributions from the fringing field effect.

Calculations were performed for fixed electrode length and channel height at $20 \mu \mathrm{m}$, using a single shelled spherical cell model [16]. Fig. $6 a$ shows the impedance sensitivity function for different frequencies, plotted against the ratio of electrode width to channel height for Design A. For any fixed ratio of $w / h$, the plot shows that the impedance sensitivity decreases with increasing frequency, as expected from dielectric theory [16], because of the dielectric dispersion. At each discrete frequency, the $S$ decreases with increasing $w / h$. In contrast [31], there is no optimal ratio of the electrode width to channel height. These results show that the impedance change is greater for larger volume fractions, that is smaller detection volume (smaller electrode width for fixed length and channel height), as the size of the cell is fixed. Fig. $6 a$ indicates that highest sensitivity is achieved when the detection volume is comparable with the volume of the cell, that is maximum volume fraction. However, for small electrodes, the interfacial impedance (electrical double layer) can limit sensitivity in the low frequency range. Figs. $6 b-c$ show the similar sensitivity analysis for Design B, showing the same trend as for Design A - a small electrode width and gap distance gives a high volume fraction (for a fixed particle size). For large volume fractions, the situation is more complicated because the particle is larger than the measuring electrodes. Comparing the magnitude of $S$ for Designs A and B, then for identical geometrical parameters Figs. $6 a-c$ show that the impedance sensitivity for Design B is much lower than for Design A. This is because the electric field from the parallel facing electrodes is confined into a smaller detection volume than the coplanar electrodes.

\section{Conclusion}

In this paper, we have presented a general method for deriving the electric field using SCM mapping. We solved the electric field distribution in a microfluidic cytometer for two different electrode arrangements. Compared with previous work, the derivations presented in the paper are systematic, and the expressions are much simpler to use. The effects of geometrical parameters on the electric field distribution have been discussed and the analytical expressions have been verified using numerical simulations. We have proposed a simple method to compare the sensitivity for the two electrode designs. We conclude that the sensitivity is not related to the ratio of $w / h$ per se, but is proportional to the volume fraction. For the same geometrical parameters, the parallel facing cytometer design is more sensitive than the coplanar electrode cytometer. The sensitivity model is however preliminary; the model is only valid for a static cell placed in the centre of the system and for low volume fractions. The system response could be evaluated in more detail by modelling a moving particle as it travels along an arbitrary path through the system.

\section{Acknowledgment}

This work was funded in part by the University of Southampton Life Sciences Initiative and Le Fond
National Suisse pour la Recherche (Bourse pour jeune chercheurs).

\section{References}

1 Green, N.G., Sun, T., Holmes, D., and Morgan, H.: 'Impedance based flow sensor'. Proc. Microtechnologies for the New Millennium, SPIE, 2005, vol. 5836, pp. 634-641

2 Collinsa, J., and Lee, A.P.: 'Microfluidic flow transducer based on the measurement of electrical admittance', Lab Chip, 2004, 4, pp. 7-10

3 Choi, J.W., Oh, K.W., Thomas, J.H., Heineman, W.R., Halsall, H.B., Nevin, J.H., Helmicki, A.J., Hendersona, H.T., and Ahna, C.H.: 'An integrated microfluidic biochemical detection system for protein analysis with magnetic bead-based sampling capabilities', Lab Chip, 2002, 2, pp. 27-30

4 Cruz, J.M., and Garcia-Diego, F.J.: 'Dielectrophoretic motion of oblate spheroidal particles. Measurements of motion of red blood cells using the Stokes method', J. Phys. D, Appl. Phys., 1998, 31, pp. $1745-1751$

5 Hughes, M.P., and Morgan, H.: 'Dielectrophoretic characterization and separation of antibody-coated submicrometer latex spheres', Anal. Chem., 1999, 71, pp. 3441-3445

6 Morgan, H., and Green, N.G.: 'AC electrokinetics: colloids and nanoparticles' (Research Studies Press, Ltd, Baldock, Hertfordshire, England, 2003)

7 Fu, A.Y., Spence, C., Scherer, A., Arnold, F.H., and Quake, S.R.: 'A microfabricated fluorescence-activated cell sorter', Nat. Biotechnol., 1999, 17, pp. 1109-1111

8 Gawad, S., Schild, L., and Renaud, Ph.: 'Micromachined impedance spectroscopy flow cytometer for cell analysis and particle sizing', Lab Chip, 2001, 1, pp. 76-82

9 Voldman, J., Gray, M.L., Toner, M., and Schmidt, A.: 'A microfabrication-based dynamic array cytometer', Anal. Chem., 2002, 74, pp. 3984-3990

10 Gawad, S., Cheung, K., Seger, U., Bertsch, A., and Renaud, Ph.: 'Dielectric spectroscopy in a micromachined flow cytometer: theoretical and practical considerations', Lab Chip, 2004, 4, pp. 241-251

11 Wang, M.M., Tu, E., Raymond, D.L., Yang, J.M., Zhang, H., Hagen, N., Dees, B., Mercer, E.M., Forster, A.H., Kariv, I., Marchand, P.J., and Butler, W.: 'Microfluidic sorting of mammalian cells by optical force switching', Nat. Biotechnol., 2005, 23, pp. 83-87

12 Huh, D., Gu, W., Kamotani, Y., Groberg, J.B., and Takayama, S.: 'Microfluidics for flow cytometric analysis of cells and particles', Physiol. Meas., 2005, 26, R73-R98

13 Cheung, K., Gawad, S., and Renaud, Ph.: 'Impedance spectroscopy flow cytometer: on-chip label-free cell differentiation', Cytometry Part A, 2005, 65A, pp. 124-132

14 Holmes, D., Morgan, H., and Green, N.G.: 'High throughput particle analysis: combing dielectrophoretic particle focusing with confocal optical detection', Biosens. Bioelectron., 2006, 21, pp. 1621-1630

15 Holmes, D., Sandison, M.E., Green, N.G., and Morgan, H.: 'On-chip high-speed sorting of micron-sized particles for high-throughput analysis', IEE Proc., Nanobiotechnol., 2005, 152, pp. 129-135

16 Morgan, H., Tao, S., Holmes, D., Gawad, S., and Green, N.G.: 'Single cell dielectric spectroscopy', J. Phys. D, Appl. Phys., 2007, 40, pp. 61-70

17 Wang, X.-B., Huang, Y., Burt, J.P.H., Markx, G.H., and Pethig, R.: 'Selective dielectrophoretic confinement of bioparticles in potential energy wells', J. Phys. D, 1993, 26, pp. 1278-1285

18 Wang, X., Wang, X.-B., Becker, F.F., and Gascoyne, P.R.C.: 'A theoretical method of electric field analysis for dielectrophoretic electrode arrays using Green's theorem', J. Phys. D, 1996, 29, pp. $1649-1660$

19 Garcia, M., and Clague, D.S.: 'The 2D electric field above a planar sequence of independent strip electrodes', J. Phys. D, 2000, 33, pp. $1747-1755$

20 Clague, D.S., and Wheeler, E.K.: 'Dielectrophoretic manipulation of macromolecules: The electric field', Phys. Rev. E, 2001, 64, p. 026605

21 Morgan, H., Izquierdo, A.G., Bakewell, D., Green, N.G., and Ramos, A.: 'The dielectrophoretic and travelling wave forces generated by interdigitated electrode arrays: analytical solution using Fourier series', J. Phys. D, 2001, 34, pp. 1553-1561

22 Chuang, D.E., Loire, S., and Mezic, I.: 'Closed-form solutions in the electrical field analysis for dielectrophoretic and traveling wave inter-digitated electrode arrays', J. Phys. D, 2003, 36, pp. 3073-3078

23 Green, N.G., Ramos, A., and Morgan, H.: 'Numerical solution of the dielectrophoretic and traveling wave forces for interdigitated electrode arrays using the finite element method', J. Electrost., 2002, 56, pp. $235-254$

24 Bruschi, P., Nannini, A., Pieri, F., Raffa, G., Vigna, B., and Zerbini, S.: 'Electrostatic analysis of a comb-finger actuator with SchwarzChristoffel conformal mapping', Sens. Actuators A, 2004, 113, pp. $106-117$ 
25 Markovic, M., Jufer, M., and Perriard, Y.: 'Analyzing an electromechanical actuator by Schwarz-Christoffel mapping', IEEE Trans. Mag., 2004, 40, pp. 1858-1863

26 Collin, R.E.: 'Field theory of guided waves' (McGraw-Hill Book Company, Inc., New York, 1960)

27 Carlsson, E., and Gevorgian, S.: 'Conformal mapping of the field and charge distributions in multilayered substrate CPWs', IEEE Trans. Microw. Theory Technol., 1999, 47, pp. 1544-1552

28 Gevorgian, S., Berg, H., Jacobsson, H., and Lewin, T.: 'Application notes - basic parameters of coplanar-strip waveguides on multilayer dielectric/semiconductor substrates, Part 1: high permittivity superstrates', IEEE Microw. Mag., 2003, 4, pp. 60-70

29 Hong, J., Yoon, D.S., Kim, S.K., Kim, T.S., Kim, S., Pak, E.Y., and No, K.: 'AC frequency characteristics of coplanar impedance sensors as design parameters', Lab Chip, 2005, 5, pp. 270-279

30 Linderholm, P., and Renaud, Ph.: 'Comment on "AC frequency characteristics of coplanar impedance sensors as design parameters" by Jongin Hong, Dae Sung Yoon, Sung Kwan Kim, Tae Song Kim, Sanghyo Kim, Eugene, Y. Pak and Kwangsoo No, Lab Chip, 2005, 5, 270', Lab Chip, 2005, 5, pp. 1416-1417

31 Linderholm, P., Seger, U., and Renaud, Ph.: 'Analytical expression for electric field between two facing strip electrodes in microchannel', IEE Electron. Lett., 2006, 42, pp. 145-147

32 Demierre, N., Braschler, T., Linderholm, P., Seger, U., Lintel, H.V., and Renaud, Ph.: 'Characterization and optimization of liquid electrodes for lateral dielectrophoresis', Lab Chip, 2007, 7, pp. 355-365

33 Schinzinger, R., and Laura, P.A.A.: 'Conformal mapping: methods and applications' (Dover Publications, Inc., Mineola, New York, 2003)

34 Gradshteyn, I.S., and Ryzhik, I.M.: 'Table of integrals, series and products' (Academic Press, San Diego, New York, 1994, 5th edn.)

35 Byrd, P.F., and Friedman, M.D.: 'Handbook of elliptic integrals for engineers and physicists' (Springer-Verlag, Berlin, 1954)

36 Maxwell, J.C.: 'A treatise on electricity and magnetism' (Dover, New York, 1954)

\section{Appendices}

\subsection{Sine-transformation}

The sine-transformation used in [30-32] can be derived using SCM method [33]. The transformation planes are shown in Fig. 7. The chosen polygonal region in the $Z$-plane, has four points: $Z_{1}, Z_{2}, Z_{3}, Z_{4}$, given by $Z_{1}=h / 2$, $Z_{2}=-h / 2, \quad Z_{3}=-h / 2+j s / 2, \quad Z_{4}=-h / 2+j(s / 2+w)$. The corresponding points at the real axis of the $U$-plane are: $U_{1}=1, U_{2}=-1, U_{3}$ and $U_{4}$. The interior polygonal angles in the $Z$-plane for $Z_{1}$ and $Z_{2}$ are $\pi / 2$. The SCM integral for performing transformation from $Z$-plane to $U$-plane is given by

$$
Z=C_{1} \int^{U} \frac{\mathrm{d} U}{\sqrt{U^{2}-1}}+C_{2}=C_{3} \int^{U} \frac{\mathrm{d} U}{\sqrt{1-U^{2}}}+C_{2}
$$

where $C_{3}=-j C_{1}$. The solution of the (33) is

$$
Z=C_{3} \sin ^{-1} U+C_{2}
$$

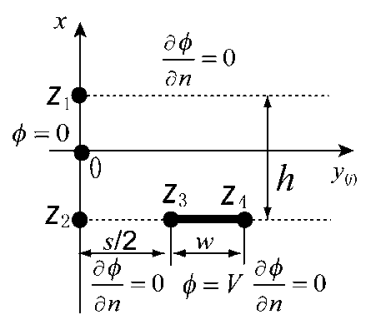

Z-Plane

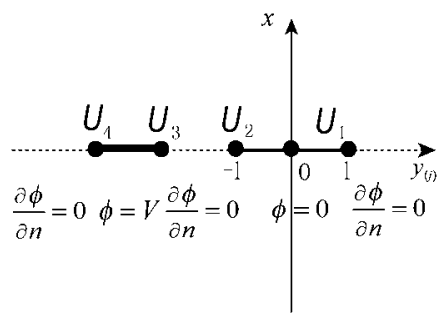

U.Plane
Fig. 7 Diagrams showing the two different planes for the sinetransformation: physical plane (Z-plane) and Model plane (U-plane)

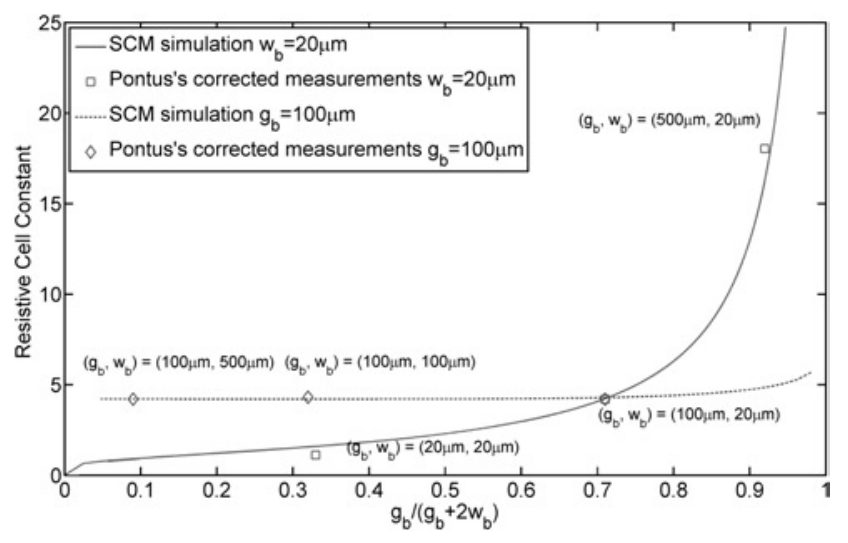

Fig. 8 Comparison of our SCM solution with the corrected experimental data from Hong et al. [29] as published in the work of Linderholm et al. [30], showing good agreement with the resistive cell constant for Design $B$

Applying the corresponding values for the boundary points, the solution becomes

$$
Z=\frac{h}{\pi} \sin ^{-1} U
$$

which gives the sine-transformation

$$
U=\sin \left(\frac{\pi Z}{h}\right)
$$

with the value of $U_{3}$ and $U_{4}$, which give the position of the electrode in $U$-plane.

$$
\begin{aligned}
U_{3}= & -\cosh \left(\frac{\pi s}{2 h}\right) \\
U_{4} & =-\cosh \left(\frac{\pi(s+2 w)}{2 h}\right)
\end{aligned}
$$

\subsection{Cell constant in Design B}

In order to compare our solutions with Hong's et al. [29] measurement results and Linderholm's [30] corrected simulation results, the resistive cell constants is calculated for different electrode width and the gap, using MATLAB. The results are shown in Fig. 8. The lines are simulations obtained form the SCM solutions, dots are the results of Hong et al., corrected by Linderholm et al. [30]. Fig. 8 shows excellent agreement with Fig. 2 of [30], validating our analytical solutions.

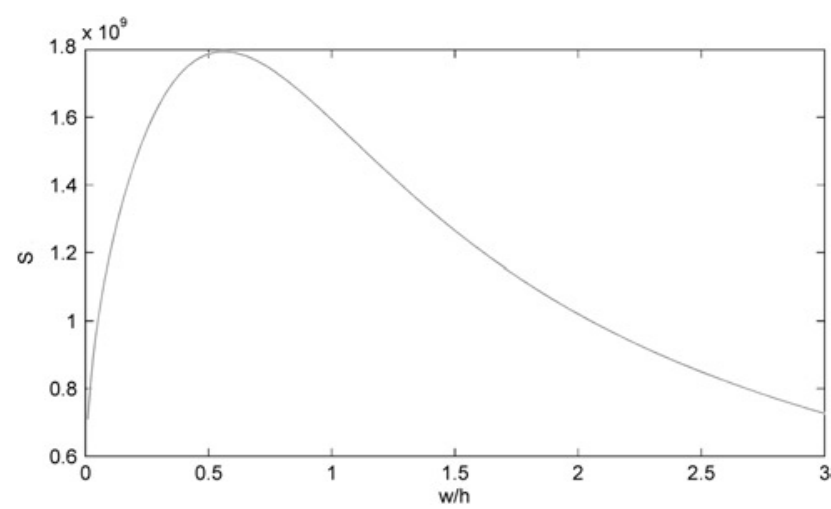

Fig. 9 Sensitivity curve as defined by Linderholm et al. [31], calculated using (39), showing the maximum value at $w / h \simeq 0.561$ This result is identical to that presented in [31], except that the maximum value in our plot is $\sim 1.8 \times 10^{9}\left(\mathrm{~V}^{2} \mathrm{~m}^{-2}\right)$ 


\subsection{Sensitivity}

Linderholm et al. [(8) in [31]] defined impedance sensitivity as

$$
S=\frac{\rho|j|^{2}}{\iint_{\text {microchannel }} \rho|j|^{2}}=\frac{R \rho\left|E_{Z} / \rho\right|^{2}}{U_{\text {applied }}^{2}}
$$

Substituting (4) of [31] into (38), and assuming $U_{\text {applied }}=1 \mathrm{~V}$, the sensitivity expression reduces to

$$
S=\left[\frac{K\left(k^{2}\right)}{K\left(1-k^{2}\right)}\right]\left|E_{Z}\right|^{2}
$$

where $K\left(k^{2}\right) / K\left(1-k^{2}\right)$ is the resistive cell constant and $E_{Z}$ is the electric field, all of which depend on the geometry. This expression was used to plot the 'sensitivity' for different ratios of electrode width to channel height, indicating a maximum at $w / h \simeq 0.561$. Plotting (39) using the electric field solution derived in our paper gives the same shape of curve as shown in Fig. $3 b$ of [31], with a maximum at $w / h \simeq 0.561$. With $h=20 \mu \mathrm{m}, E_{Z} \simeq 10^{4} \mathrm{~V} / \mathrm{m}$ in the centre of the channel, giving a maximum value for $S$ of $10^{9}\left(\mathrm{~V}^{2} \mathrm{~m}^{-2}\right.$ ) (see Fig. 9). This value should be compared with the $S_{\max } \simeq 2.5 \%$, quoted in [31]. However, the authors did not explain how their curves were scaled. 